Psychotherapeut 2010 · 55:370-372

DOI 10.1007/s00278-010-0769-0

Online publiziert: 27. August 2010

(c) Springer-Verlag 2010

Wolfgang Schneider

Klinik und Poliklinik für Psychosomatik und Psychotherapeutische Medizin, Zentrum für Nervenheilkunde, Universität Rostock

\title{
Schwerpunktheft zum Thema Standards und Methoden der Begutachtung
}

verweisen, die hohe Zahl an Arbeitsunfähigkeitszeiten wegen dieser Erkrankungen und zum anderen in dem Sachverhalt, dass diese Krankheiten seit einer Reihe von Jahren zu Renten wegen verminderter Erwerbsfähigkeit führen. Aber auch bei den privaten Berufsunfähigkeitsrenten und bei Schadensregulierungen von Unfallversicherungen nehmen die psychischen und psychosomatischen Erkrankungen bzw. Unfallfolgen eine große Bedeutung ein.

Es hat den Anschein, als ob in wirtschaftlich brisanten Zeiten, die durch einen wachsenden Druck auf den Arbeitsmarkt (hohe Arbeitslosigkeit und drohende Arbeitslosigkeit) sowie durch die vielfach berichtete Verschärfung der Arbeitsbedingungen gekennzeichnet sind, die Tendenz zur Ausbildung von psychischen und psychosomatischen Krankheiten ansteigt. Diese Entwicklung könnte einen gewichtigen Grund dafür darstellen, dass Begutachtungen von psychischen Störungen in unterschiedlichen Kontexten mehr und mehr an Bedeutung gewonnen haben.

Neben dem, sicherlich nicht anzweifelbaren Sachverhalt, dass psychische und psychosomatische Erkrankungen in unserer Lebenswelt mit ihrer Komplexität, Schnelligkeit, sozialen Unsicherheit und den damit verbundenen hohen Anforderungen an die Fähigkeit des Einzelnen zur Selbstregulierung und sozialen Adaptation, zahlenmäßig zugenommen haben, ist die Relevanz, die diese in gutachterlichen Kontexten einnehmen, sicherlich auch in ihrer „Unschärfe“ auf der diagnostischen Ebene begründet. Psychische und psychosomatische Störungen im Gesamt ihrer Symptome bieten sich in besonderer Weise als Begründungsmodelle für die Beanspruchung von Renten oder Schadensansprüchen an. Aber auch chronische körperliche Erkrankungen sind durch psychosomatische Komplikationen gekennzeichnet, aus denen sich entsprechende Rechtsansprüche grundsätzlich ableiten lassen. So werden vielfach bei entsprechenden Rentenverfahren, wenn somatische Erkrankungen und damit verbundene Funktionsbeeinträchtigungen einen Rentenanspruch nicht rechtfertigen, psychische und psychosomatische Gründe oder Komplikationen als Begründung für rentenrechtlich relevante Leistungseinschränkungen vorgebracht. Dies gilt insbesondere für chronische Schmerzerkrankungen, bei denen auf der Ebene des körperlichen Substrates nur ungenügende Befunde erhoben worden sind, die eine relevante Leistungseinschränkung nicht rechtfertigen.

Im sozialen Entschädigungsrecht, dem Unfallrecht oder dem Asylrecht spielen die posttraumatischen Belastungsstörungen bei Begutachtungsfragestellungen eine wichtige Rolle. Auch hier stellt die "Weichheit“ oder „Unschärfe“ der diagnostischen Prozesse und der gutachterlichen Entscheidungsfindung ein 
relevantes Problem für alle Beteiligten inkl. der Gerichtsbarkeit dar, wobei sich die Konstellation oft noch durch ethische oder politische Aspekte (wie Gerechtigkeit) verschärft.

Auf diesem Hintergrund ist nur zu gut verstehbar, dass für die Begutachtung der Leistungsfähigkeit oder etwaiger Kausalitätszusammenhänge bei psychischen und psychosomatischen Erkrankungen die inhaltlichen Konzepte und die methodischen Standards so elaboriert und so klar wie möglich definiert und beschrieben werden müssen. Insbesondere die Kriterien, die zur Entscheidungsfindung führen, müssen explizit und transparent sein.

Im Rahmen von Gutachten stellt sich regelhaft die Aufgabe, etwaige Tendenzen zur Symptomaggravation oder der Simulation von Beschwerden und deren Folgen abzuklären. Eine Reihe von einschlägigen Untersuchungen legt nahe, dass sich entsprechende Tendenzen nicht selten bei zu Begutachtenden finden. Nach Schätzungen weisen bis zu 50\% der zu Begutachtenden eine relevante Tendenz zur Aggravation ihrer Beschwerden bzw. zur Dissimulation ihrer Leistungsfähigkeit auf.

Die Beurteilung etwaiger systematischer Aggravationstendenzen muss auf einer umfassenden Analyse unterschiedlicher Informationen (z. B. Interviewverhalten, Befunde der klinischen Untersuchung, testpsychologische Ergebnisse und Testverhalten) aufbauen. Nur unter der Berücksichtigung und Integration unterschiedlicher Hinweise auf das Vorliegen von Aggravationstendenzen im konkreten Fall lässt sich diese Problematik einigermaßen angemessen beantworten.

Ein grundlegendes Problem der Validität der Begutachtung der Leistungsfähigkeit, aber auch der Kausalitätsfrage ist in der Komplexität dieser Konstrukte begründet. Die berufliche Leistungsfähigkeit ist durch soziale Umweltfaktoren (Verfügbarkeit und Art der Arbeit), iatrogene Faktoren, die ggf. über wiederholte - nicht oder nur eingeschränkt indizierte diagnostische und therapeutische - Maßnahmen zur Chronifizierung führen, und soziale Netzwerkvariablen (die z. B. den Rentenwunsch unterstützen) mitbeeinflusst. Individuelle Faktoren, zu denen Persönlichkeitsvariablen, konflikt- haft gefärbte Motive und (Krankheits-) Verarbeitungsmechanismen sowie dysfunktionale Lernprozesse gehören, spielen bei der Entstehung und Entwicklung der psychischen oder psychosomatischen Krankheit sowie bei der Herausbildung des subjektiven Leistungskonzeptes und vermögens eine wichtige Rolle. Insbesondere die individuelle Bewertung des Leistungsvermögens wird durch motivationale Variablen bestimmt. Diese können jedoch sowohl bewusst z. B. als rationale Bewertung der persönlichen Chancen, unter spezifischen Arbeitsbedingungen eine angemessene und persönlich umsetzbare Tätigkeit zu erhalten, als auch unbewusst (z. B. durch maladaptive regressive Prozesse infolge von psychischen Störungen) beeinflusst werden. Entlang meiner Erfahrungen als Gutachter finden sich bei vielen begutachteten Personen sowohl unbewusst als auch bewusst motivierte aus der Sicht des Gutachters als nichtangemessen bewertete - subjektive Leistungskonzepte.

Die Begutachtung unterschiedlicher Fragestellungen bei psychischen und psychosomatischen Erkrankungen ist aufgrund der nur eingeschränkten Verfügbarkeit von angemessen Methoden und Entscheidungskriterien, der komplexen Determiniertheit des Begutachtungsgegenstandes und der „Weichheit“ der zu bewertenden Variablen und Befunde also insgesamt als problematisch anzusehen.

Bei der Entwicklung einer Begutachtungssystematik müssen unterschiedliche diagnostische Merkmalsbereiche berücksichtigt und beurteilt werden. Für einzelne diagnostische Kategorien mögen genügend valide Außenkriterien (z. B. für die Messung der Psychopathologie oder psychischer Funktionen) zur Verfügung stehen, jedoch existieren keine Messinstrumente, die das komplexe Merkmal beruflicher Leistungsfähigkeit valide beurteilen lassen. Auch Assessment-Center, die im Schwerpunkt die körperliche Leistungsfähigkeit messen, haben nur eine eingeschränkte Aussagekraft für diese Fragestellung.

Darüber hinaus sollen nicht nur Querschnittbewertungen (Wie ist die Leistungsfähigkeit jetzt einzuschätzen?) vorgenommen werden, sondern die berufliche Leistungsfähigkeit sollte immer auch in Bezug auf ihre zukünftige Entwicklung bewertet werden. So sind eigentlich prospektive Untersuchungen notwendig, die Aussagen über den Verlauf der Leistungsfähigkeit über die Zeit und über unterschiedliche Lebens- und Arbeitsbedingungen erlauben. Letztlich wird es jedoch so sein, dass die Entwicklung der Leistungsfähigkeit durch eine Vielzahl von intervenierenden Variablen, wie z. B. das Fortschreiten von Chronifizierungsprozessen infolge der Rentengewährung, beeinflusst wird. Die Ergebnisse von prospektiven Studien wären darüber hinaus sicherlich auch in einem relevanten Ausmaß durch Aggravationstendenzen der Untersuchten belastet. So bleibt zu resümieren, dass die Begutachtungen in dem hier diskutierten Problemfeld auch zukünftig durch eine Reihe von Unschärfen charakterisiert sein werden.

In dem vorliegenden Schwerpunktheft werden von Schneider et al. in zwei Arbeiten konzeptionelle und methodische Vorgehensweisen bei der Leistungsbegutachtung diskutiert und dargestellt, die im Rahmen einer von der Dr.-Karl-WilderStiftung des Gesamtverbandes des Versicherers geförderten multizentrischen Studie erarbeitet worden sind. Dieser Ansatz wird auch in den aktuell erarbeiteten Leitlinien zur Begutachtung der Leistungsfähigkeit im Auftrag der einschlägigen Fachgesellschaften verfolgt.

Freyberger $u$. Widder zeigen in ihrem Aufsatz die psychiatrisch-psychosomatischen Grundzüge sowie die juristischen Rahmenbedingungen bei der Begutachtung von Kausalitätsfragen auf. Darüber hinaus werden die besonderen Probleme der Begutachtung von Individuen mit Somatisierungsstörungen durch Schickel und Henningsen et al. erörtert. Die Begutachtung psychischer und psychosomatischer Erkrankungen erfordert die Berücksichtigung unterschiedlicher methodischer Ansätze. Einen wichtigen Zugang stellen dabei psychologische Testverfahren dar. Die Reichweite und Grenzen dieser Methoden werden von Dohrenbusch u. Merten prägnant und anwendungsnah diskutiert; in einem gesonderten Aufsatz werden die Möglichkeiten psychologischer Testverfahren zur Abklärung von Tendenzen zur Symptomaggravation dargelegt. Mit diesem Schwerpunktheft wer- 
den die aktuellen inhaltlichen und methodischen Standards der Leistungsbegutachtung, aber auch ihre Reichweite und Grenzen umfassend konzeptionell, methodisch und anwendungsnah dargestellt. Wir gehen davon aus, dass, bei allen Beschränkungen, die hier vorgestellten Ansätze zukünftig einen Qualitätsstandard für die Begutachtung psychischer und psychosomatischer Erkrankungen darstellen werden.

\section{Korrespondenzadresse}

Prof. Dr. Dr. Wolfgang Schneider Klinik und Poliklinik für Psychosomatik und Psychotherapeutische Medizin, Zentrum für Nervenheilkunde, Universität Rostock Gehlsheimer Str. 20, 18147 Rostock wolfgang.schneider@med.uni-rostock.de

\section{Schwerpunktthemen}

Das Herausgebergremium der Zeitschrift Psychotherapeut lädt Autorinnen und Autoren ein, an den geplanten Schwerpunkten mitzuarbeiten und geeignete Manuskripte einzureichen. Diese werden dem üblichen Reviewverfahren unterzogen. Bitte schicken Sie Ihren Beitrag an die Redaktion:

Regine.Karcher-Reiners@springer.com

Heft 2/11: Erinnern und Vergessen (Deadline 18.11.2010)

Heft 3/11: Chronische Erschöpfung (Deadline 14.1.2011)

Heft 4/11: Psychoonkologie (Deadline 18.3.2011)

Heft 5/11: Psychische Gesundheit (Deadline 25.5.2011)

Heft 6/11: Arbeit (Deadline 26.7.2011)

Heft 1/12: OPD (Deadline: 17.9.2011)

Heft 2/12: Themata der Lindauer Psychotherapiewochen 2011 (Deadline 18.11.2011)

Heft 3/12: Bedeutung des Therapeuten für den Therapieerfolg (Deadline 14.1.2012) 